\title{
Vértebra limbus cervical
}

\section{Cervical Vertebra Limbus}

\author{
Florencio Jiménez-Martín ${ }^{1} \quad$ Enrique Vázquez-Muñoz ${ }^{2}$ \\ ${ }^{1}$ Servicio de Medicina Física y Rehabilitación, Hospital Universitario La \\ ${ }^{2}$ Servicio de Radiología, Hospital Universitario La Paz, Madrid, España
}

\section{David Hernández-Herrero ${ }^{1}$}

Rev Argent Radiol 2020;84:75-76.

Estimados editores,

La vértebra limbus es un hallazgo radiológico consistente en un fragmento óseo localizado en el margen de uno de los ángulos de un cuerpo vertebral.

Se presenta el caso de una vértebra limbus de localización infrecuente, en columna vertebral cervical, que puede dar lugar a un diagnóstico erróneo, dadas sus características radiológicas.

Presentamos el caso de un varón de 28 años de edad, que consultó por dolor facial de cuatro años de evolución. Había sido diagnosticado como dolor facial atípico. No contaba con antecedentes patológicos de interés. Presentaba un dolor facial izquierdo irradiado a la región cervical izquierda, sin clínica neurológica. Los episodios de dolor no eran constantes $\mathrm{y}$, cuando aparecían, tardaban varias horas en desaparecer. La intensidad máxima del dolor, medida por la escala EVA (escala visual analógica), era de 5/10. No tomaba analgésicos, debido a que toleraba bien el dolor. En la exploración física se apreciaba una movilidad cervical conservada, la exploración neurológica fue normal, solo encontrando dolor en la palpación del fascículo superior del músculo trapecio izquierdo. La proteína $C$ reactiva fue normal $(<0,5 \mathrm{mg} / \mathrm{dl})$. La tomografía computarizada (TC) cerebral fue normal.

En la radiografía simple lateral de columna cervical se observa, como hallazgo casual, un defecto óseo de forma triangular en el borde anterosuperior de la vértebra $\mathrm{C} 5$, con márgenes bien definidos (-Fig. 1A). En la resonancia magnética (RM) cervical, también se muestra una separación de un fragmento de hueso triangular en la misma localización (-Fig. 1B).

La vértebra limbus es un hallazgo radiológico de prevalencia desconocida y significado incierto. ${ }^{1}$

La herniación intraósea anterior del núcleo pulposo vertebral, produciendo una vértebra limbus, fue descrita por primera vez por Christian Georg Schmorl ${ }^{2}$ en 1927. Es
Address for correspondence Florencio Jiménez-Martín, MD, Servicio de Medicina Física y Rehabilitación, Hospital Universitario La Paz, Calle Julio Palacios 29, Escalera B, 13-D, Madrid, España (e-mail: fljmartin@gmail.com). el resultado de una herniación marginal anterior intraósea del núcleo pulposo de una vértebra durante la etapa de desarrollo del esqueleto, causando la separación de un fragmento de hueso triangular, que se puede confundir con la epífisis anular vertebral no fusionada, diferenciándose de la hernia de Schmorl en que ésta es una hernia más central en el platillo vertebral superior o inferior. ${ }^{1-3}$

La imagen radiológica es característica y generalmente no se necesitan otras exploraciones complementarias para hacer el diagnóstico.

En algunas ocasiones, ese fragmento óseo puede sufrir un desplazamiento superior o anterior sin llegar a provocar afectación del espacio discal ni de los cuerpos vertebrales adyacentes. ${ }^{2}$ En los adultos, el fragmento óseo tiene una
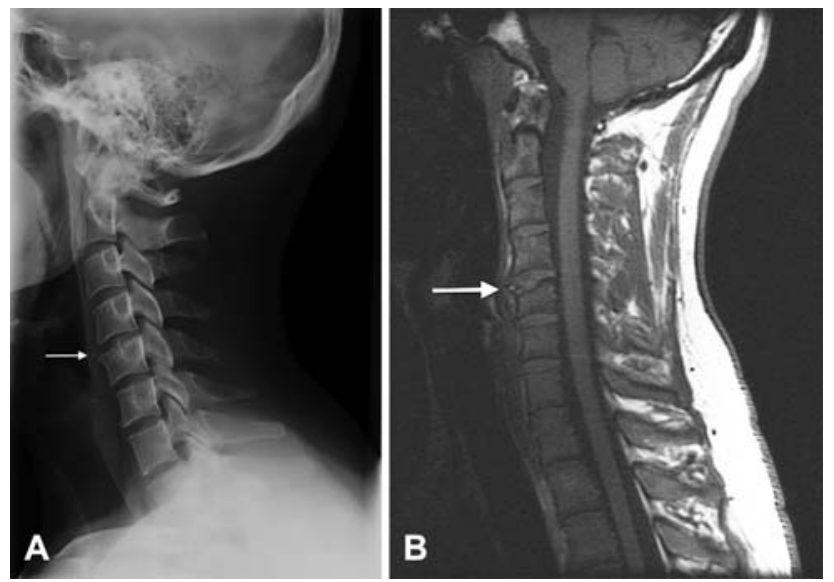

Fig. 1 (A) La radiografía lateral de la columna cervical muestra un pequeño fragmento óseo, con márgenes bien definidos, sobre el ángulo anterosuperior del quinto cuerpo vertebral cervical (flecha). (B) RM cervical sagital potenciada en T1 que muestra una separación de un fragmento de hueso triangular (flecha) en el ángulo anterosuperior del quinto cuerpo vertebral cervical. received January 29, 2020 accepted January 29, 2020
DOI https://doi.org/ $10.1055 / \mathrm{s}-0040-1702994$ ISSN 1852-9992.
Copyright $\odot$ 2020, Sociedad Argentina de Radiología. Publicado por Thieme Revinter Publicações Ltda., Rio de Janeiro, Brazil. Todos los derechos reservados.

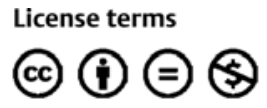


morfología triangular y nítida y unos bordes escleróticos, mientras que en niños y adolescentes los márgenes del fragmento óseo pueden ser irregulares y mal definidos, simulando un proceso lítico, como una infección o un tumor. ${ }^{2}$

La localización más frecuente es la columna lumbar, el borde anterosuperior seguido del anteroinferior del cuerpo vertebral. $^{1}$

La vértebra limbus causa síntomas inespecíficos como dolor de espalda, espasmos musculares y radiculopatía. A menudo, se diagnostica erróneamente como una fractura, una infección, una hernia intraesponjosa de Schmorl o un tumor, lo que puede dar lugar a realizar pruebas diagnósticas, o incluso procesos invasivos, innecesarios. ${ }^{3,4}$

Para realizar el diagnóstico puede ser suficiente la radiografía simple, pero en los casos dudosos se pueden realizar técnicas de imagen complementarias para confirmarlo y descartar otras patologías. Entre las técnicas utilizadas están la discografía, en desuso al ser un procedimiento invasivo, la TC, actualmente es la prueba diagnóstica de elección, y la RM, que permite una mejor visualización de los tejidos blandos. ${ }^{1}$

En la mayoría de los casos es un hallazgo casual y, por lo general, no necesita tratamiento. Los pacientes sintomáticos son tratados de forma conservadora con analgésicos, relajantes musculares y rehabilitación, reservándose el tratamiento quirúrgico, necesario en muy pocas ocasiones, para aquellos casos en los que el dolor es recurrente e incapacitante. ${ }^{2,5}$ Los resultados del tratamiento quirúrgico son variables y entre las técnicas utilizadas en los casos de vértebra limbus posterior están la laminectomía total y la extirpación del fragmento óseo. ${ }^{5}$

\section{Responsabilidades Éticas}

Los autores garantizan que la investigación realizada en el trabajo se ha obtenido con el consentimiento informado y se han respetado las pautas éticas de la institución a la que pertenecen y de la Declaración de Helsinki de 1975, con la revisión de octubre de 2000.

Conflicto de Intereses

Los autores declaran no tener ningún conflicto de intereses.

\section{Bibliografía}

1 Carrasco Cubero C, Coelho Achega DG, Férnández Gil MÁ, Álvarez Vega JL. Mechanical low back pain as a presentation of anterior limbus vertebra. Reumatol Clin. 2017;13(03):176-177

2 Horneros Torres J, Rodríguez Muguruza S, Pérez Andrés R. Limbus vertebra. Semin Fund Esp Reumatol. 2012;13(02):62-64

3 Tuna S, Özdemir T, Öz HE. Limbus Vertebra Presenting with Inflammatory Low Back Pain: A Case Report. J Clin Diagn Res. 2016;10(03):YD01-YD02

4 Jover Llopis A, Aranda Santos B, Romero Perera JJ, Umanes Alvarez MI. Vértebra limbus múltiple: ¿un hallazgo radiológico inespecífico o causa determinante de dolor lumbar? Rehabilitación. 2018;52 (02):134-136

5 Huang PY, Yeh LR, Tzeng WS, Tsai MY, Shih TT, Pan HB, et al. Imaging features of posterior limbus vertebrae. Clin Imaging. 2012;36(06):797-802 